\title{
CubeSat's Deployable Solar Panel with Viscoelastic Multilayered Stiffener for Launch Vibration Attenuation
}

\author{
Shankar Bhattarai, ${ }^{1}$ Hongrae Kim, ${ }^{2}$ and Hyun-Ung Oh $\mathbb{D}^{1}$ \\ ${ }^{1}$ Space Technology Synthesis Laboratory, Department of Aerospace Engineering, Chosun University, 309, Pilmun-daero, Dong-gu, \\ Gwangju 61452, Republic of Korea \\ ${ }^{2}$ Soletop Co. Ltd., 409 Expo-ro, Yuseong-gu, Daejeon, Republic of Korea
}

Correspondence should be addressed to Hyun-Ung Oh; ohu129@chosun.ac.kr

Received 10 March 2020; Revised 17 July 2020; Accepted 24 July 2020; Published 11 August 2020

Academic Editor: Mohammad Tawfik

Copyright (c) 2020 Shankar Bhattarai et al. This is an open access article distributed under the Creative Commons Attribution License, which permits unrestricted use, distribution, and reproduction in any medium, provided the original work is properly cited.

\begin{abstract}
Ensuring the structural safety of a deployable solar panel under a severe launch vibration environment is one of the important factors for a successful CubeSat mission. A CubeSat's deployable solar panel proposed in this study is effective to guarantee the structural safety of solar cells by attenuating launch loads owing to the superior damping characteristic achieved by a multilayered stiffener with viscoelastic acrylic tapes. The demonstration model of $3 \mathrm{U}$ CubeSat's deployable solar panel was fabricated and tested to validate the effectiveness of the proposed design. The basic dynamic characteristics of the solar panel were measured through free-vibration tests according to the various layers of the stiffener. Moreover, the characteristics of the deployed solar panel were measured and investigated under various temperatures to predict its capability under in-orbit operation. The effectiveness of the proposed design for launch vibration attenuation was demonstrated through qualification level sine and random vibration tests.
\end{abstract}

\section{Introduction}

A CubeSat is a cube-shaped nanoclass satellite platform having a volume of $1000 \mathrm{~cm}^{3}$ and a mass of $1.33 \mathrm{~kg}$ per a standard size of one unit (1 U) [1]. Owing to the low cost and short period for construction and launch, this miniaturized satellite platform has received considerable attention in the space engineering field. The CubeSats were primarily envisioned for low-cost educational [2] and scientific [3] missions in universities and research institutions. However, in recent, the trend of the CubeSat development is changing from traditional low-volume production to industry-scale high-volume production. This trend has facilitated the realization of more advanced and challenging missions based on CubeSat constellations, such as near real-time earth observation [4], worldwide internet service [5], and meteorological monitoring [6].

The $3 \mathrm{U}$ or larger sized CubeSats, equipped with more advanced and miniaturized instruments such as cameras, antennas, sensors, and electronics, have been increasingly developed to improve mission capability of a CubeSat platform [4-6]. However, it has caused an increase in the electrical power required to realize the advanced CubeSat mission. One technical solution to satisfy this increasing power demand is the adoption of a deployable solar panel taking into account the limited available area to install the solar cells owing to the extreme spatial constraint of a CubeSat structure. Nowadays, many CubeSat vendors produce various configurations of flight-proven deployable solar panels based on the printed circuit board (PCB) substrate, which is advantageous for rapid fabrication and provides ease of electrical interconnection between the solar cells [7]. For stowing and releasing those deployable panels, hold and release mechanisms (HRMs) based on a burn wire triggering method are widely utilized owing to their simplicity, low cost, and ease of mechanism reset $[8,9]$. These mechanisms usually provide a mechanical constraint on the solar panel during launch by tightening the nylon wire, which is then cut by heating the nichrome wire or burn resistor to release the panel in orbit [10]. 
The deployable solar panel mounted on the CubeSat is subjected to severe launch vibration environments. The dynamic deflection of a solar panel under vibration causes stress on the solar cells mounted on the panel by the bounded junction, which could ultimately lead to a crack or fracture in those cells. In the case of previously developed deployable solar panels for CubeSat applications, the mechanical design strategy for the deflection minimization was to increase the panel stiffness by applying additional stiffeners made up of various materials like aluminium, carbon-fiber-reinforced plastic, or fiberglass laminate $[11,12]$. However, this strategy typically led to an increase in the mass and development cost of the solar panel, which might be disadvantageous for CubeSats with an extremely restricted budget. In addition, an acceleration response of the stiffened solar panel might be increased in conjunction with the mass increase. These adverse effects result in a higher tensile force acting on the wire cutting mechanisms that constraint the panel during launch. Moreover, that force would be further increased with an increase in the solar panel's size. However, most of the burn wire triggering-type mechanisms have a limited holding capability because they hold the panel relying mainly on the nylon wire with low stiffness and strength. This means that the potential risk of the mechanism failure in a launch environment could be increased owing to the excessive mechanical loading acting on the wire by panel excitation. As an alternative solution in regard to the above issue, multiple numbers of mechanisms can be applied to provide additional mechanical fixation points on the solar panel. However, if one of them fails to release the panel in-orbit, the satellite might not sufficiently produce the electrical power and it would become a critical issue for the entire mission. Furthermore, the application of multiple mechanisms to a single panel could reduce the available area for solar cell attachment.

Another potential technical problem related to the deployable solar panel is an oscillation in the deployed panel induced by satellite attitude maneuvering, because it causes a rigid body motion in the satellite, which might degrade its pointing stability in-orbit [13]. This oscillation problem might be unavoidable if the panel size becomes larger owing to the low flexible mode of the solar panel. Additionally, the conventional technologies for reducing panel oscillation that have been widely applied for large-class satellites, such as rotary damper or deployable truss link, are extremely difficult to apply them for CubeSats due to the spatial limitations allowed for the deployable appendages. These facts indicate that an alternative way for reducing in-orbit panel oscillation might be necessary for advanced CubeSat missions.

Considering the trend that the CubeSat's solar panels become larger for advanced missions, the aforementioned technical problems related to the vibrations under launch and in-orbit environments shall be solved. This is the starting point of this study. In this study, we focus on a multilayered PCB with viscoelastic acrylic tapes for attenuation of the launch and in-orbit vibrations. The applications of viscoelastic materials for vibration attenuation have been widely studied in space engineering fields owing to the simplicity and cost-effectiveness. For example, Minesugi and Onoda [14] investigated the damping mechanism of a polyimide tape with viscous lamina for space applications. Torisaka and Yamakawa [15] proposed a passive vibration damping approach for the solar panel of a small satellite by considering viscoelastic materials and lightweight aluminium stiffeners. Kwon et al. [16] developed a multilayered blade-type passive vibration isolator with double-sided viscoelastic adhesive tapes to attenuate the launch and in-orbit vibrations for a spaceborne cryogenic cooler. Furthermore, Steinberg [17] reported that constrained layers of viscoelastic material could be effective for reducing dynamic displacements and stresses in vibrating beam and plate structures.

In the present study, we proposed a CubeSat's PCB-based deployable solar panel employing a multilayered stiffener, interlaminated with viscoelastic acrylic tape, for ensuring the structural safety of the solar cells under severe launch vibration environments. An important advantage of the proposed solar panel is that it provides exceptionally high damping, which cannot be achieved with the currently used materials. In addition, this damping characteristic may also be advantageous to rapidly attenuate the oscillation of the deployed solar panel during an in-orbit operation. In this study, a demonstration model of a 3 U CubeSat's deployable solar panel was fabricated and tested to validate the effectiveness of the proposed design. The damping ratio and the natural frequency of the solar panel were investigated by conducting free-vibration tests with various layers of stiffeners. Additionally, the effect of temperature on the damping performance and stiffness of the solar panel was investigated through that test. Furthermore, to validate the effectiveness of the design in terms of the launch load attenuation on the solar panel, sine and random vibration tests were performed at a qualification level. The test results demonstrated that the design approach proposed in this study is effective to attenuate the launch loads for ensuring the structural safety of the solar panel in a launch environment.

\section{Solar Panel with Viscoelastic Multilayered Stiffener}

2.1. Design Drivers. The size of CubeSat has recently been increased to $3 \mathrm{U}, 6 \mathrm{U}, 12 \mathrm{U}$, and $27 \mathrm{U}$ for achieving more challenging and sophisticated missions through the CubeSat platform that has led to the use of deployable solar panels to meet on-board power demand. However, it involves the problem of excessive dynamic displacement of the panel during a launch environment that could cause delamination or stress in the solar cells. To overcome this issue, most of the previous strategies have focused on increasing the stiffness of the panel by applying additional stiffeners made up of various materials such as aluminium and fiberglass laminate or using a carbon-fiber-reinforced plastic (CFRP) panel and honeycomb panel. For example, ISISpace [18] developed deployable solar panels, where a thin PCB solar panel with a thickness of $0.18 \mathrm{~mm}$ was stiffened using an aluminium panel. Park et al. [11] developed a PCB-based 6 U CubeSat's deployable solar panel stiffened by high-pressure fiberglasslaminated G10 material. Besides, Lim et al. [12] developed a 6 U CubeSat's deployable solar panel based on an aluminium 
honeycomb panel instead of the PCB to ensure a stiffness requirement specified by the launch provider. In the case of stiffener strategy, the rigidity of the stiffener is the key point to increase the eigenfrequency of the panel. However, it involves an inevitable increase in mass and acceleration response of the stiffened panel that could produce an undesirable burden on HRM, especially the ones based on the nylon wire cutting mechanisms generally used in CubeSat applications. The CFRP and honeycomb panels are relatively light in weight and exhibit high stiffness, thus commonly been used in aerospace applications wherever high strength to weight ratio and rigidity are required. However, those structures are expensive and thick in size that has made them less practical for advanced missions of CubeSat platform due to the internal restraint edge gap on the polypicosatellite orbital deployer (P-POD), $6.5 \mathrm{~mm}$ [1] or $10 \mathrm{~mm}$ [19] for the solar panel accommodation and development cost limitations.

As an alternative solution in regard to the above issues, multiple numbers of HRMs could be applied to provide additional mechanical fixation points on the solar panel, although it could increase system complexity and reduce the available area for solar cell accommodation. These facts indicate that a new strategy is required to minimize the dynamic deflection of the solar panel for the structural safety of the solar cells as well as to reduce the potential risk of mechanical failure in HRM. Thus, in this study, we proposed a CubeSat's PCB-based deployable solar panel employing a thin multilayered stiffener, interlaminated with viscoelastic acrylic tapes, as a new strategy to guarantee structural safety of solar cells by attenuating the launch loads on the panel under launch environment.

2.2. Design Description. To demonstrate the effectiveness of stiffeners with viscoelastic acrylic tapes for launch load attenuation of the solar panel, a $3 \mathrm{U}$ sized solar panel as shown in Figure 1 was fabricated. The demonstration model of the dummy solar panel is mainly comprised of a PCB panel, thin PCB stiffeners, and viscoelastic acrylic tapes. The PCB panel is made out of FR4 material with dimensions $320 \mathrm{~mm} \times 82$ $\mathrm{mm} \times 1.6 \mathrm{~mm}$, which provides a mechanical interface for the integration of stiffeners and solar cells. Thin PCB stiffeners as shown in Figure 1 of thickness $0.5 \mathrm{~mm}$ were attached to the rear surface of the PCB panel by a doublesided $3 \mathrm{M}^{\mathrm{TM}} 966$ acrylic tape [20]. The basic principle for attenuating the launch load on the panel is based on the resistive deformation characteristics of laminated adhesive tapes subjected to shear deformation such that the constrained viscoelastic layer dissipates maximum vibration energy. The $3 \mathrm{M}^{\mathrm{TM}} 966$ acrylic tape manufactured by the $3 \mathrm{M}$ company is a high-temperature acrylic adhesive with low outgassing properties that meets the National Aeronautics and Space Administration (NASA) low volatility specification criteria of ASTM E596. Thus, it has been used in aerospace applications for bonding purposes. The detailed specifications of the materials used in the solar panel are presented in Table 1.

The manufacturing process of the PCB stiffener is simple; the four center rectangular blocks as well as a hinge and the HRM portion of the PCB were intentionally cut out for mass optimization that also allows access to the rear side of the solar panel. Furthermore, the longitudinal and the transverse structural shape of the stiffeners effectively mitigates longitudinal and torsional oscillation of the panel with the combination of viscoelastic acrylic tape. The stiffener attachment process is considerably simple, although intensive care should be given to control the uniform bonding strength distribution in workmanship. The unsymmetrical attachments of the acrylic tape would increase prestress and delamination in the multilayered stiffener. Therefore, four guide holes were considered on the four edge corners of the stiffeners and the PCB panel for effortless integration of stiffeners on the panel. The total thickness of the solar panel after integration of the five layers of stiffener on the PCB panel is $4.5 \mathrm{~mm}$ that allows an additional margin of $5.5 \mathrm{~mm}$ lateral edge gap on P-POD for a dynamic clearance [19]. Figure 2 shows the front and rear view of the demonstration model of the $3 \mathrm{U}$ solar panel. Table 2 summarizes the mass budget of the demonstrated model of the solar panel. The total mass of the solar panel proposed in this study is $141 \mathrm{~g}$.

\section{Experimental Evaluation of Viscoelastic Multilayered Stiffener for Vibration Attenuation on the Solar Panel}

3.1. Basic Dynamic Characteristics. The vibration attenuation of the constrained layer damping with viscoelastic materials is highly dependent on geometric parameters such as crosssectional area, shape, and thicknesses of the viscoelastic and constraining layers, because the energy dissipation actions mainly resulted from the shear deformation behavior between the interlaminated surfaces $[22,23]$. To investigate the basic dynamic characteristics of the solar panel, such as damping performance and stiffness, with respect to the number of adhesive tape attachment conditions, free-vibration tests were performed. The following three cases were considered: solar panel without a stiffener and solar panels with 3 and 5 layers of the stiffener, in order to clarify the characteristics of the design in each case. In the above-mentioned cases, the free-vibration tests were carried out at ambient room temperature of $25^{\circ} \mathrm{C}$ for the solar panel under boundary condition that both hinge and HRM hole interfaces are rigidly clamped, which corresponds to a launch stowed configuration of the solar panel. The roving hammer method was used to excite the solar panel to its free vibration. An accelerometer sensor to obtain the time domain response was attached at the center of the panel to measure the frequency responses of the solar panel. The experimental test result shown in Figure 3 demonstrates the time histories of the free-vibration response of the solar panels. Figure 4 shows the power spectral density (PSD) acceleration responses of the solar panel with various numbers of interlaminated stiffeners. The results show that the vibration of the solar panel can be effectively suppressed by employing the stiffeners with viscoelastic acrylic tapes.

Figure 5 shows the $1^{\text {st }}$ eigenfrequency and damping ratio variation of the solar panel according to the application of the stiffener. The damping ratios of the solar panel after the 


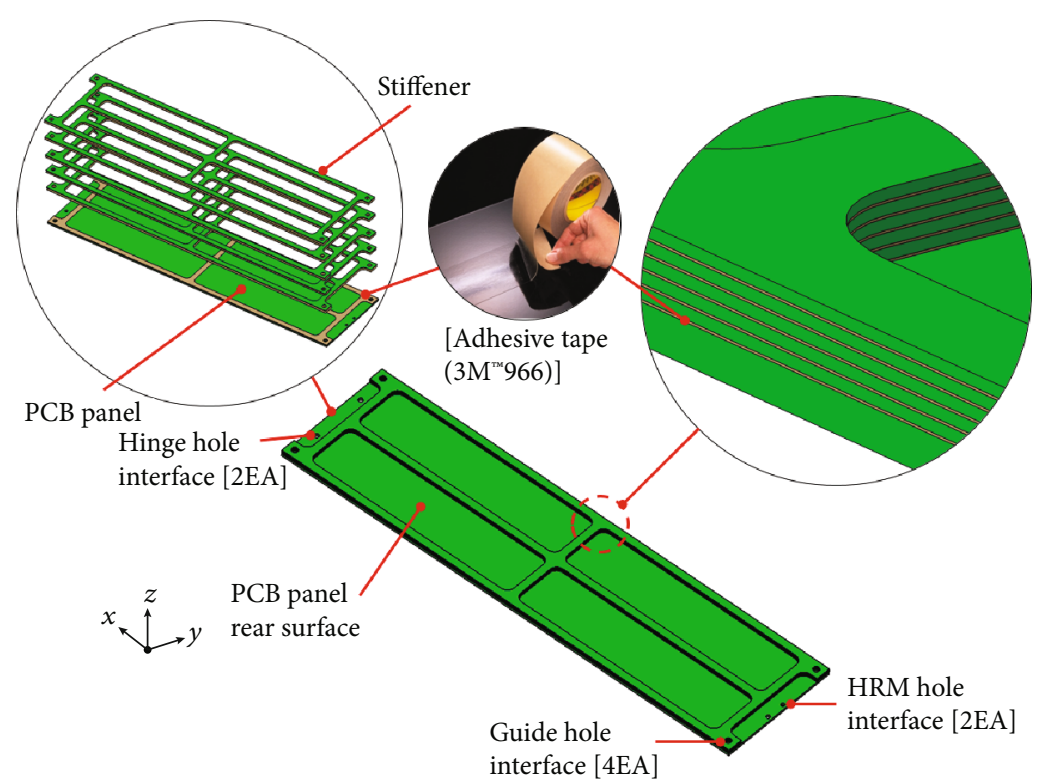

FIgURE 1: Illustrative design configuration of a 3 U CubeSat's deployable solar panel.

TABLE 1: Specification of materials used in the solar panel.

\begin{tabular}{|c|c|c|}
\hline Item & Details & Value \\
\hline \multirow{9}{*}{$\begin{array}{l}3 \mathrm{M}^{\mathrm{TM}} 966 \text { adhesive } \\
\text { transfer tape }[20]\end{array}$} & Manufacturer & 3M Company \\
\hline & Adhesive material & Acrylic \\
\hline & Color & Transparent \\
\hline & Thickness (mm) & 0.06 \\
\hline & $\begin{array}{l}\text { Thermal conductivity } \\
\left(\text { at } 41^{\circ} \mathrm{C}\right)(\mathrm{W} / \mathrm{m} / \mathrm{K})\end{array}$ & 0.178 \\
\hline & $\begin{array}{l}\text { Coefficient of thermal } \\
\text { expansion }\left(\mathrm{ppm} /{ }^{\circ} \mathrm{C}\right)\end{array}$ & 1.99 \\
\hline & Allowable temperature $\left({ }^{\circ} \mathrm{C}\right)$ & $-40 \sim 232$ \\
\hline & $\begin{array}{l}\text { Adhesive strength (to steel) } \\
\qquad(\mathrm{N} / 100 \mathrm{~mm})\end{array}$ & 159 \\
\hline & $\begin{array}{c}\text { Outgassing } \\
(\%, \text { TML/CVCM })\end{array}$ & $0.93 / 0.01$ \\
\hline \multirow{4}{*}{ FR4 [21] } & Elastic modulus $(\mathrm{Pa})$ & $18.73 \times 10^{9}$ \\
\hline & Poisson's ratio & 0.136 \\
\hline & Density $\left(\mathrm{kg} / \mathrm{m}^{3}\right)$ & 1850 \\
\hline & $\begin{array}{l}\text { Thermal conductivity } \\
(\mathrm{W} / \mathrm{m} / \mathrm{K})\end{array}$ & 0.29 \\
\hline
\end{tabular}

application of 3 and 5 layers of the viscoelastic stiffener were, respectively, 0.019 and 0.061 , which is higher by factors of 9.5 and 30.5 as compared to 0.002 that was measured in the nonstiffener solar panel. Furthermore, the $1^{\text {st }}$ eigenfrequency calculated from the vibration periods of the solar panel without a stiffener and with 3 and 5 layers of the stiffener was $36.70 \mathrm{~Hz}, 63.52 \mathrm{~Hz}$, and $68.81 \mathrm{~Hz}$, respectively. The result shows that the application of the viscoelastic multilayered stiffener substantially enhanced the vibration damping as well as increased the stiffness of the panel due to the fact that large shear strain and relatively tough surface roughness were

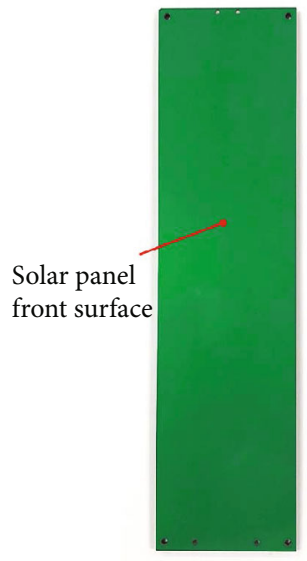

(a)

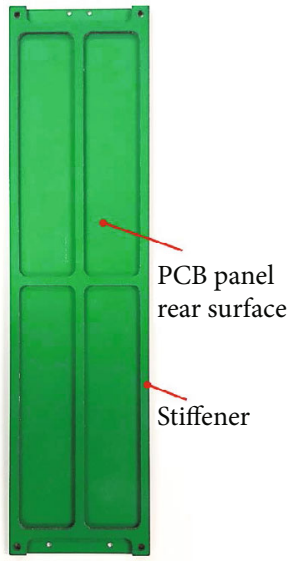

(b)
Figure 2: Demonstration model of the $3 \mathrm{U}$ solar panel: (a) front view and (b) rear view.

achieved by molecular attraction force of acrylic polymers of the viscoelastic acrylic tapes. The global bending mode is the most dominant in the dynamic displacement of the panel even if the stiffeners are mounted on the panel although the dynamic displacement would be decreased owing to the increased stiffness of panel assembly [24-26]. The mode shape corresponding to the fundamental frequency of the solar panels remains the same. This is because the stiffness value of the stiffeners themselves is smaller than the panel, such that they do not change the original mode shape at the $1^{\text {st }}$ eigenfrequency of the panel although the modes at higher frequency might be changed in some extent. The stiffness difference between the solar panel without a stiffener and with the implementation of 3 layers of the stiffener is relatively higher than that observed between the 3 and 5 layer cases. This indicates that increasing the number of stiffeners 
TABLE 2: Mass budget of the demonstration model of the solar panel.

\begin{tabular}{lc}
\hline Item & Mass $(\mathrm{g})$ \\
\hline PCB panel & 93 \\
5 layers of PCB stiffeners & 45 \\
$3 \mathrm{M}^{\mathrm{TM}}$ 966 acrylic adhesive tapes & 3 \\
\hline Total & 141 \\
\hline
\end{tabular}

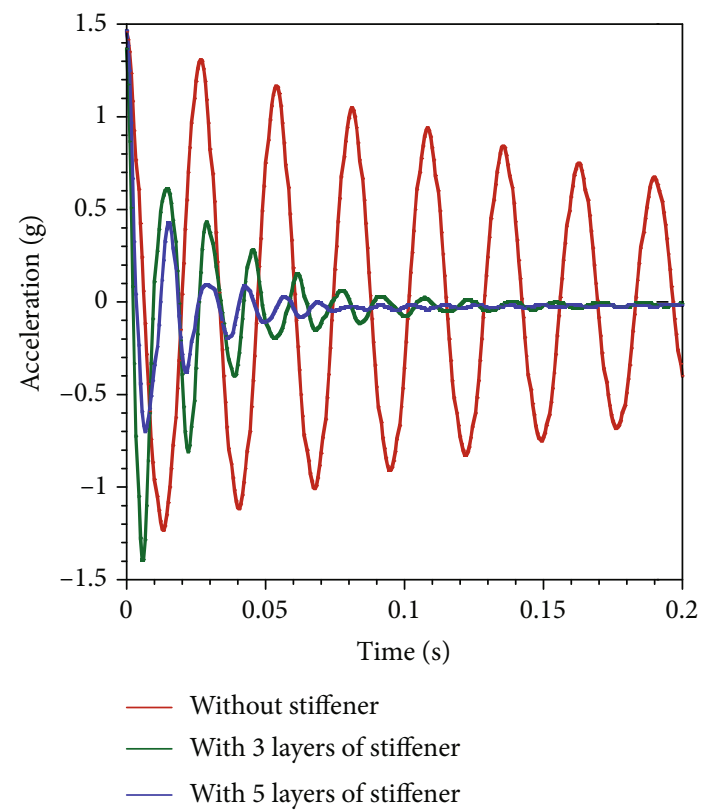

FIGURE 3: Free-vibration test results of the solar panels with various numbers of stiffeners.

raised the panel rigidity to some extent, however, not as a linear function of stiffener numbers. However, this kind of asymmetry was not observed in the damping performance of the panel because the amount of energy dissipated in a viscoelastic layer depends on the magnitude of shear strain in that layer. Thus, the number of viscoelastic stiffener layers should be taken into account as a design parameter by considering the panel mass, stiffness, and lateral thickness imposed in the system requirements.

For CubeSat applications, where rapid slew maneuvers for the acquisition of a target point are required, the interaction of deployable solar panels with the attitude control system could generate a rigid body motion that can significantly degrade performance in terms of pointing accuracy and acquisition time. With the current trend of large deployable solar panels with low-frequency modes to meet the power requirement in CubeSat design, the residual vibration at the end of attitude maneuver as well as slewing time increases that significantly degrades the performance requirement of future advanced missions. However, the proposed design of the solar panel could also substantially suppress the vibration of the solar panel during in-orbit operations that may overcome the above issues to some extent. Nevertheless, the properties of viscoelas-

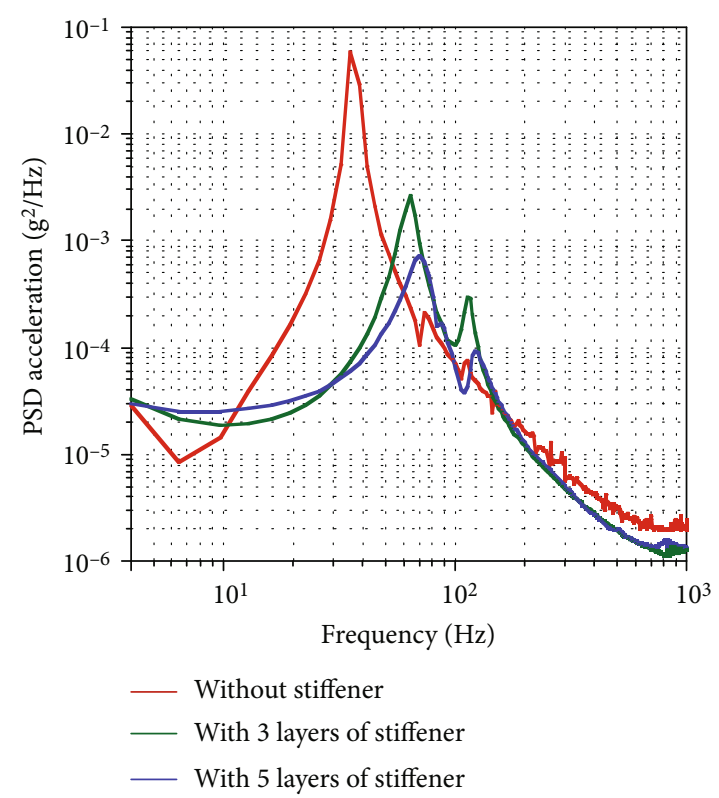

FIgURE 4: The PSD acceleration responses of the solar panel with various numbers of interlaminated stiffeners.

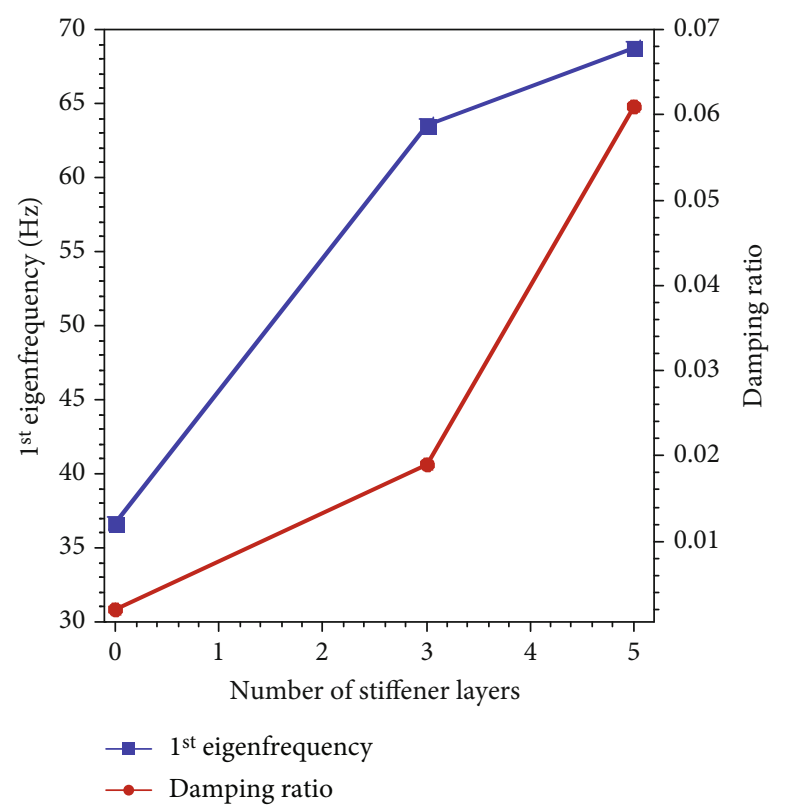

FIGURE 5: $1^{\text {st }}$ eigenfrequency and damping ratio of the solar panel according to the number of stiffener layers.

tic materials used in constrained layer damping are generally much more sensitive to temperature that could affect the dynamic characteristics of the solar panel according to the temperature condition. Thus, free-vibration tests of solar panels considering in-orbit deployed configuration were performed at various temperature ranges in the thermal chamber. The objective of this test was to investigate the characteristic variations of the deployed solar panel, such as damping performance and stiffness in various temperature conditions for 


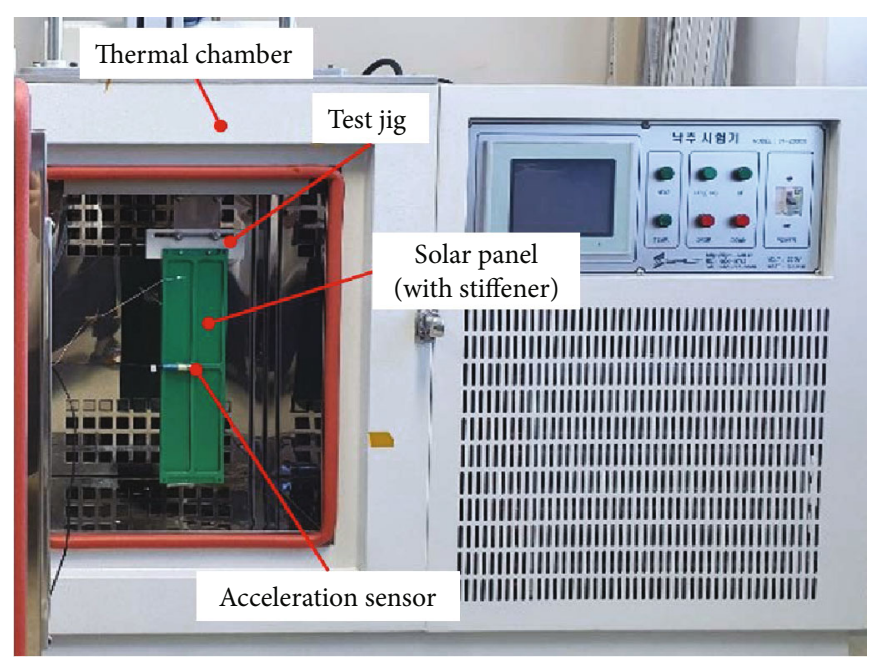

FIGURE 6: Free-vibration test setup configuration of the solar panel in a thermal chamber.

predicting its design effectiveness under in-orbit operation. Thus, the specified temperature range was set as $-20^{\circ} \mathrm{C}$ to $60^{\circ} \mathrm{C}$, which covers the expected glass-transition temperature range of $3 \mathrm{M}^{\mathrm{TM}} 966$ acrylic tape, although the allowable temperature range is $-40^{\circ} \mathrm{C}$ to $232^{\circ} \mathrm{C}$. In order to ensure the uniform temperature distribution throughout the specimen panel, the thermal dwell time at a particular test temperature was set to one hour. Figure 6 shows the free-vibration test setup configuration of the solar panel in a thermal chamber. The solar panel was cantilevered on the test jig to acquire the characteristic variation in the low-frequency range that could help to predict design effectiveness under in-orbit deployed configuration. To measure the frequency responses of the panel during the free-vibration test, an accelerometer sensor was attached at the center of the solar panel.

Figure 7 shows the $1^{\text {st }}$ eigenfrequency of solar panels in accordance with temperature variations. The result indicates that the stiffness of a solar panel varies with environmental temperature due to the structural phase transition effect of the material. The solar panel with 5 layers of stiffener exhibited relatively higher stiffness over all temperature conditions, compared to the other cases. This is explained by the fact that nearly stable adhesive strength is maintained on thin multiple stiffeners by high-temperature acrylic tapes such that the stiffeners used as a constraining layer accumulate the equivalent stiffness of the panel.

Figure 8 shows the damping ratio of the solar panels in accordance with the temperature variations. Likewise to stiffness, the damping ratio of the solar panel is also profoundly dependent on the temperature because the energy dissipation action is accomplished mainly by the shear strain of viscoelastic layers. At lower temperatures, so-called glassy region, the viscoelastic acrylic tape instigates to behave as an elastic material; thus, the damping performance of the panel remains lower owing to the high storage modulus. As the temperature increases, the damping ratio of the solar panel is relatively higher because the viscoelastic acrylic layer dissipates maximum vibration energy owing to the shear deformation until the glass transition temperature is reached [20]. Meanwhile, this is the point of maximum shear strain

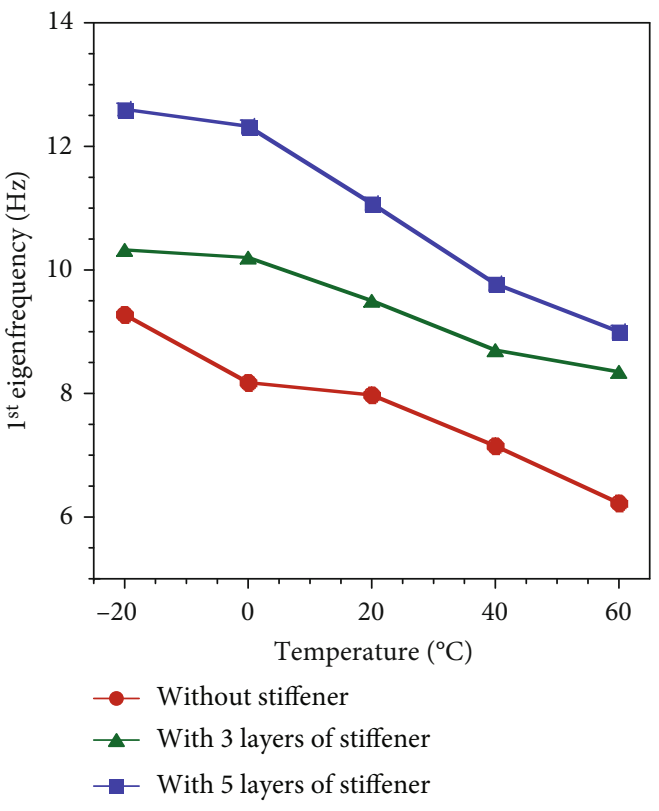

FIGURE 7: $1^{\text {st }}$ eigenfrequency of the solar panels under various temperature conditions.

and further increase in temperature results decreasing the solar panel's damping ratio owing to the viscous molecules of adhesive tape instigating a rubbery state above the glass transition temperature. Where both the storage modulus and the loss modulus of viscoelastic material remain small, minimum energy dissipation is therefore obtained from the cyclic shear deformation of adhesive tapes. As shown in the result, all panels showed similar damping behavior with temperature variation, although the solar panel employing 5 layers of stiffener exhibited a relatively higher damping magnitude than other cases due to the cumulative shear strain contributed by the increased cross-sectional area of adhesive tapes.

3.2. Launch Vibration Test. To evaluate the structural safety and damping performance of the proposed design of a solar 


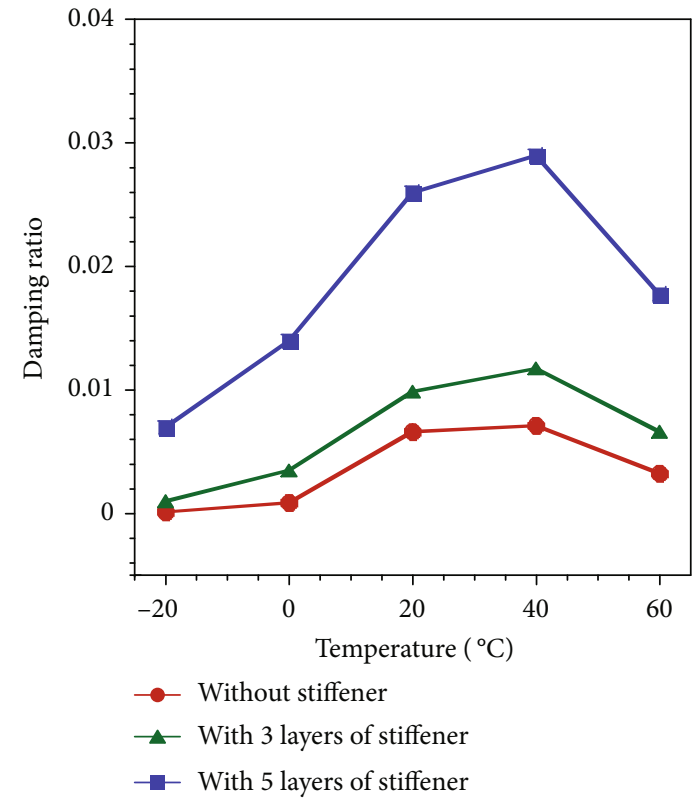

FIGURE 8: Damping ratio of the solar panels under various temperature conditions.

panel in launch environments, launch vibration tests such as sine and random vibration tests were carried out under the qualification level of the launch load specified in Table 3. The main objective of these tests was to verify the design effectiveness of the highly damped solar panel system with viscoelastic acrylic tapes to ensure the structural safety of solar cells in launch vibration loads.

Figure 9 shows an example of a launch vibration test configuration of the solar panel along with $z$-axis excitation. The demonstration model of the solar panel was rigidly mounted on the electrodynamic vibration shaker (J260/SA7M, IMV Corp.) through the bolt fastening on the HRM and hinge hole interfaces. An accelerometer sensor was attached to the test jig to control the input vibration load. The output acceleration responses of the solar panel were measured by the accelerometer attached at the center of the solar panel. The test was performed at an ambient room temperature of $18^{\circ} \mathrm{C}$. The structural safety of the solar panel is validated by comparing the variation of the $1^{\text {st }}$ eigenfrequencies of the panel obtained through low-level sine sweep (LLSS) tests performed before and after each vibration test. In order to judge the structural safety of the specimen under the launch load, the $1^{\text {st }}$ eigenfrequency variation in LLSS should be less than $5 \%$.

Figure 10 shows the sine vibration test results of solar panels along with $z$-axis excitation. The $z$-axis is the most critical axis because it induces the largest dynamic deflection of the solar panel. With respect to the maximum $2.5 \mathrm{~g}$ sine vibration input load, the maximum acceleration response of the solar panel without a stiffener was $43.72 \mathrm{~g}$ which was observed at $47.6 \mathrm{~Hz}$. Moreover, at the same input vibration load, the maximum acceleration response of the solar panels with 3 and 5 layers of stiffeners was $16.59 \mathrm{~g}$ and $11.45 \mathrm{~g}$ at $58.5 \mathrm{~Hz}$ and $73 \mathrm{~Hz}$, respectively. As seen in the results, the solar panel's damping efficiency and stiffness were substan-
TABLE 3: Summary of qualification level launch vibration test specifications.

\begin{tabular}{|c|c|c|c|c|}
\hline \multicolumn{5}{|c|}{ Sinusoidal vibration test [27] } \\
\hline Level & $\begin{array}{c}\text { Frequency } \\
(\mathrm{Hz})\end{array}$ & $\begin{array}{l}\text { Amplitude } \\
\text { (g) }\end{array}$ & \multicolumn{2}{|c|}{ Sweep rate (oct/min) } \\
\hline \multirow{3}{*}{$\begin{array}{l}0 \mathrm{~dB} \\
\text { (full level) }\end{array}$} & 5 & 1.3 & \multirow{3}{*}{\multicolumn{2}{|c|}{2}} \\
\hline & 8 & 2.5 & & \\
\hline & 100 & 2.5 & & \\
\hline \multicolumn{5}{|c|}{ Random vibration test [28] } \\
\hline Level & $\begin{array}{l}\text { Frequency } \\
\quad(\mathrm{Hz})\end{array}$ & $\begin{array}{c}\text { Amplitude } \\
\left(\mathrm{g}^{2} / \mathrm{Hz}\right)\end{array}$ & $\begin{array}{c}\text { Test } \\
\text { duration (s) }\end{array}$ & $\begin{array}{c}\text { RMS } \\
\text { acceleration } \\
\left(G_{\mathrm{rms}}\right) \\
\end{array}$ \\
\hline \multirow{4}{*}{$\begin{array}{l}0 \mathrm{~dB} \\
\text { (full level) }\end{array}$} & 20 & 0.026 & \multirow{4}{*}{120} & \multirow{4}{*}{14.10} \\
\hline & 50 & 0.16 & & \\
\hline & 800 & 0.16 & & \\
\hline & 2000 & 0.026 & & \\
\hline
\end{tabular}

tially increased with respect to the number of attached stiffeners. Among those, the solar panel with 5 layers of stiffener exhibited higher damping performance, i.e., lower amplification factor than other cases because of the increased number of viscoelastic layers that upsurge shear deformations during panel deflection.

Figure 11 shows the random vibration test results along with the $z$-axis excitation of the solar panel. The $G_{\text {rms }}$ values obtained from acceleration power spectral density (APSD) profiles of a solar panel without a stiffener and with 3 and 5 layers of the stiffener were $38.42,14.03$, and 12.63 , respectively. The output response of the solar panel with 5 layers of the stiffener was lower by a factor of 1.12 than that of the input $14.1 G_{\text {rms }}$ level. Likewise, to the above sine vibration test results, solar panels showed similar dynamic behavior under a random vibration load.

Table 4 compares the $1^{\text {st }}$ eigenfrequencies of the solar panels obtained from the LLSS tests that were performed before and after each vibration test. The tabulated LLSS result shows that the $1^{\text {st }}$ eigenfrequency shifted was within $3.42 \%$ throughout the test sequences of the panels, which is within the $5 \%$ criterion. In addition, after completion of the launch vibration tests, the visual inspection of the solar panel does not report any crack, dissociation, and plastic deformation on the stiffeners although it is not shown here. These tests and inspection results indicate that the structural safety of the proposed solar panel was successfully verified in qualification level launch environment tests.

Table 5 summarizes the relative dynamic displacement derived from the sensor response measured at the center of the solar panel during the worst excitation axis along the $z$ -direction. The maximum dynamic displacement of the solar panels without a stiffener and with 3 and 5 layers of the stiffener, under random vibration load, estimated from the threesigma value of $G_{\text {rms }}$ response was $1.39 \mathrm{~mm}, 0.13 \mathrm{~mm}$, and $0.04 \mathrm{~mm}$, respectively. The dynamic deflection of the solar panel employing 5 layers of the stiffener is significantly reduced by a factor of 34.75 as compared to the solar panel without a stiffener. This is owing to the higher vibration 


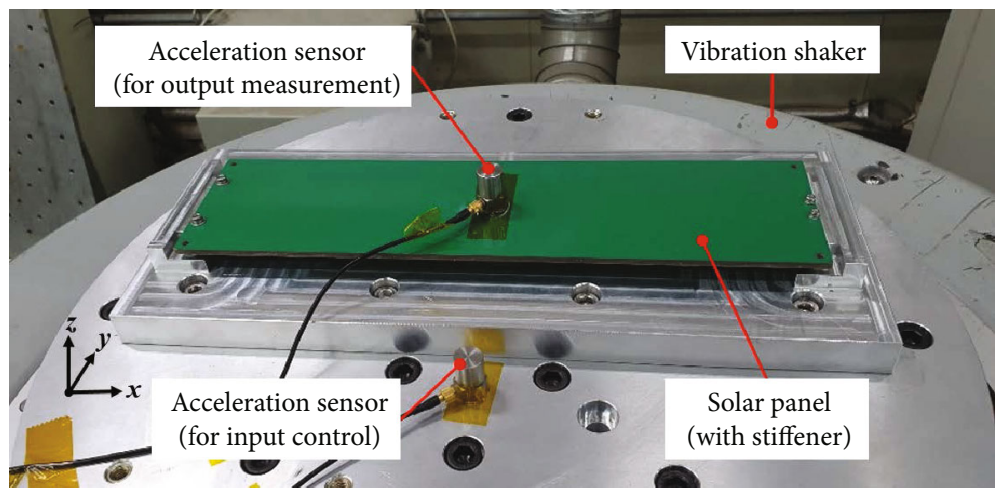

FIGURE 9: Launch vibration test setup configuration of the solar panel.

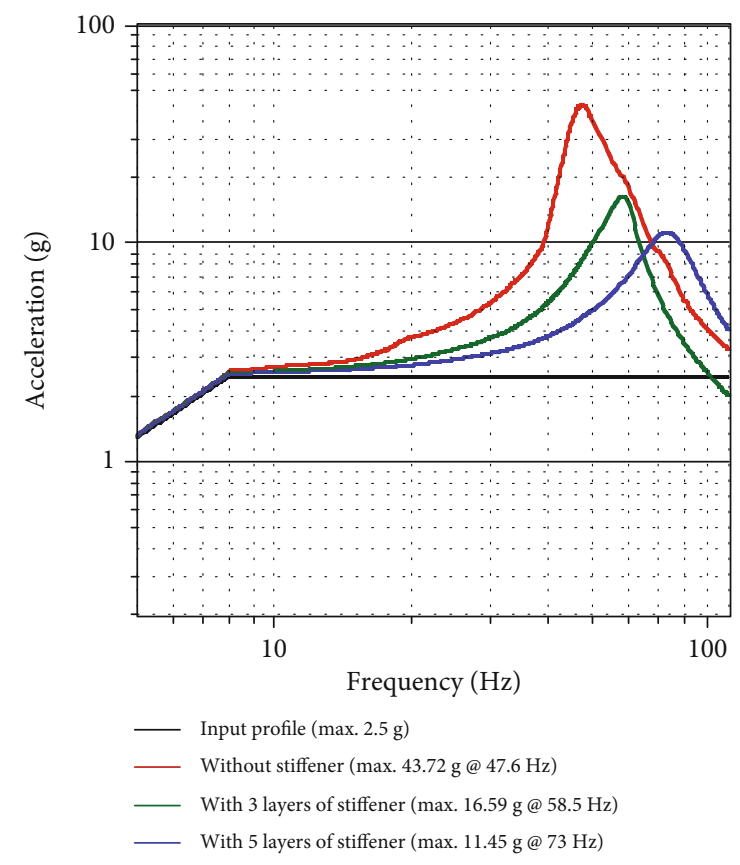

FIGURE 10: Sinusoidal vibration test results of the solar panels in the $z$-axis excitation.

attenuation resulted from the increased shear area of viscous layers of an acrylic tape. Moreover, the increased natural frequency of the panel also contributed to the reduction in dynamic displacement. Thus, a solar panel employing constrained layer damping with viscoelastic acrylic tapes proposed in this study is effective for achieving the design goals of launch load attenuation and minimization of the dynamic deflection of the panel. If this technology is applicable for $6 \mathrm{U}$ or larger configurations, the use of a single HRM makes it possible to ensure the structural safety of solar cells without reducing the available area of solar cell accommodation within the minimal increased mass of the solar panel module.

\section{Conclusion}

In this study, the effectiveness of constrained layer damping with viscoelastic acrylic tapes on the CubeSat's PCB-based

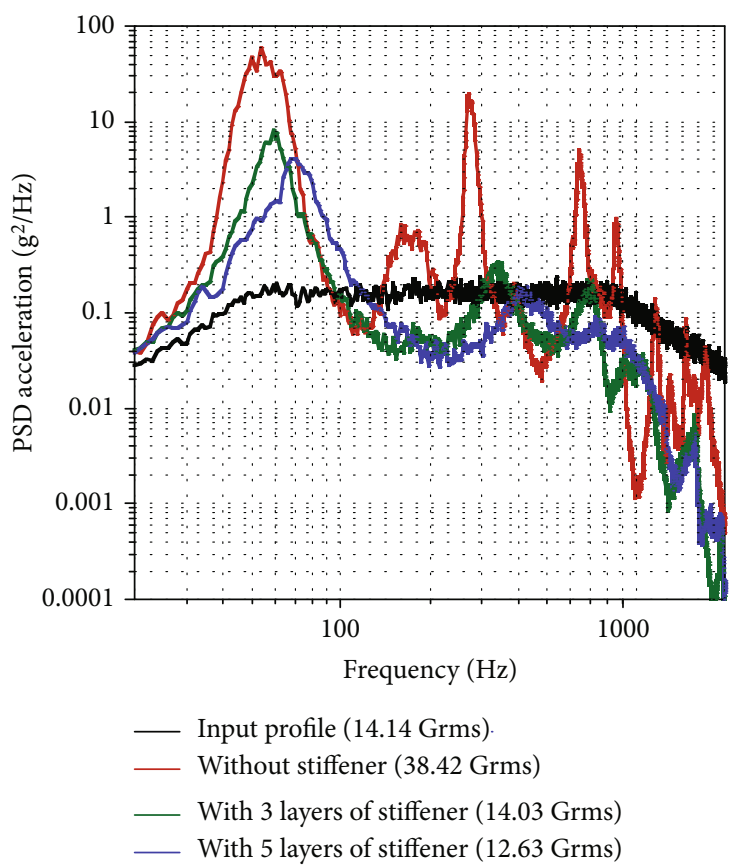

FIgURE 11: Random vibration test results of the solar panels in the $z$ -axis excitation.

deployable solar panel was evaluated to ensure the structural safety of solar cells under a launch vibration environment. A demonstration model of the $3 \mathrm{U}$ CubeSat's deployable solar panel exhibited exceptionally high damping performance owing to the excessive shear deformation achieved by the combination of multilayered stiffeners and viscoelastic tapes. The basic dynamic characteristics of the solar panel were demonstrated by free-vibration tests under various temperature conditions. The design effectiveness for attenuating the launch loads and the structural safety of the solar panel was experimentally verified through the qualification level sine and random vibration tests. The launch vibration results of the solar panel with 5 layers of stiffener demonstrated that the dynamic displacement was significantly reduced by a factor of 34.75 compared to that of the solar panel without a stiffener. In addition, the solar panel design suggested in this study could also have a considerable advantage for the rapid 
TABLE 4: LLSS test results of solar panels before and after full level vibration test.

\begin{tabular}{|c|c|c|c|c|}
\hline Test & Solar panel & Status & $1^{\text {st }}$ eigenfrequency $(\mathrm{Hz})$ & Difference (\%) \\
\hline \multirow{6}{*}{ Sine vibration } & \multirow{2}{*}{ w/o stiffener } & Before & 47.6 & \multirow{2}{*}{2.90} \\
\hline & & After & 46.2 & \\
\hline & \multirow{2}{*}{ with 3 layers of stiffener } & Before & 58.5 & \multirow{2}{*}{3.41} \\
\hline & & After & 56.5 & \\
\hline & \multirow{2}{*}{ with 5 layers of stiffener } & Before & 73.0 & \multirow{2}{*}{0.68} \\
\hline & & After & 73.5 & \\
\hline \multirow{6}{*}{ Random vibration } & \multirow{2}{*}{ w/o stiffener } & Before & 47.6 & \multirow{2}{*}{2.90} \\
\hline & & After & 46.2 & \\
\hline & \multirow{2}{*}{ with 3 layers of stiffener } & Before & 58.5 & \multirow{2}{*}{3.42} \\
\hline & & After & 56.5 & \\
\hline & \multirow{2}{*}{ with 5 layers of stiffener } & Before & 72.5 & \multirow{2}{*}{1.37} \\
\hline & & After & 73.5 & \\
\hline
\end{tabular}

TABle 5: Estimated dynamic displacement of solar panels in vibration loads.

\begin{tabular}{lcc}
\hline Test & Solar panel & $\begin{array}{c}\text { Max. displacement } \\
(\mathrm{mm})\end{array}$ \\
\hline \multirow{3}{*}{ Sine vibration } & w/o stiffener & 0.49 \\
& with 3 layers of stiffener & 0.12 \\
& with 5 layers of stiffener & 0.05 \\
\hline \multirow{3}{*}{ Random vibration } & w/o stiffener & 1.39 \\
& with 3 layers of stiffener & 0.13 \\
& with 5 layers of stiffener & 0.04 \\
\hline
\end{tabular}

attenuation of residual vibration on deployed solar panels after the slew maneuver of satellite that could minimize the performance degradation of the future space missions, where rapid slew maneuvers are required for the acquisition of a target point.

\section{Data Availability}

The data used to support the findings of this study are available from the corresponding author upon request.

\section{Conflicts of Interest}

The authors declare that there is no conflict of interest regarding the publication of this manuscript.

\section{Acknowledgments}

This research was supported by a research fund (2019) from Chosun University.

\section{References}

[1] S. Lee, A. Hutputanasin, A. Toorian, W. Lan, and R. Munakata, CubeSat design specification rev. 12, California Polytechnic State University, San Luis Obispo, USA, 2009.
[2] J. Bouwmeester and J. Guo, "Survey of worldwide pico- and nanosatellite missions, distributions and subsystem technology," Acta Astronautica, vol. 67, no. 7-8, pp. 854-862, 2010.

[3] K. Woellert, P. Ehrenfreund, A. J. Ricco, and H. Hertzfeld, "Cubesats: cost-effective science and technology platforms for emerging and developing nations," Advances in Space Research, vol. 47, no. 4, pp. 663-684, 2011.

[4] G. Santilli, C. Vendittozzi, C. Cappelletti, S. Battistini, and P. Gessini, "CubeSat constellations for disaster management in remote areas," Acta Astronautica, vol. 145, pp. 11-17, 2018.

[5] S. C. Burleigh, T. De Cola, S. Morosi, S. Jayousi, E. Cianca, and C. Fuchs, "From connectivity to advanced internet services: a comprehensive review of small satellites communications and networks," Wireless Communications and Mobile Computing, vol. 2019, 17 pages, 2019.

[6] A. Poghosyan and A. Golkar, "CubeSat evolution: analyzing CubeSat capabilities for conducting science missions," Progress in Aerospace Sciences, vol. 88, pp. 59-83, 2017.

[7] Small spacecraft technology state of the art - NASA, Small Spacecraft Systems Virtual Institute, Ames Research Center, Moffett Field, California, 2018.

[8] S. Bhattarai, H. Kim, S. H. Jung, and H. U. Oh, "Development of pogo pin-based holding and release mechanism for deployable solar panel of CubeSat," International Journal of Aerospace Engineering, vol. 2019, 13 pages, 2019.

[9] GomSpace, NanoPower DSP-GOMspaceJune 2020, https:// gomspace.com/UserFiles/Subsystems/datasheet/gs-dsnanopower-dsp-11.pdf.

[10] T. Y. Park, S. H. Kim, H. Kim, and H. U. Oh, "Experimental investigation on the feasibility of using spring-loaded pogo pin as a holding and release mechanism for CubeSat's deployable solar panels," International Journal of Aerospace Engineering, vol. 2018, Article ID 4854656, 10 pages, 2018.

[11] T. Y. Park, B. G. Chae, and H. U. Oh, "Development of 6 U CubeSat's Deployable Solar Panel with Burn Wire Triggering Holding and Release Mechanism," International Journal of Aerospace Engineering, vol. 2019, 13 pages, 2019.

[12] L. S. Lim, T. D. V. Bui, K. S. Low et al., "VELOX-II: challenges of developing a $6 \mathrm{U}$ nanosatellite," AIAA SPACE Conference and Exposition, 2016, pp. 1-11, Long Beach, California, September 2016. 
[13] J. Wang, D. Li, and J. Jiang, "Optimal variable amplitudes input shaping control for slew maneuver of flexible spacecraft," Journal of Guidance, Control, and Dynamics, vol. 40, no. 12, pp. 3255-3263, 2017.

[14] K. Minesugi and J. Onoda, "Passive vibration suppression using thin tape with viscous lamina," in AIAA 36th Structures Structural Dynamics and Materials Conference, pp. 200-206, New Orleans, LA, U.S.A, April 1995.

[15] A. Torisaka and H. Yamakawa, "Optimum vibration control design of a light weight structure in wide frequency domain," Journal of Environment and Engineering, vol. 6, no. 2, pp. 328-339, 2011.

[16] S. C. Kwon, M. S. Jo, D. H. Ko, and H. U. Oh, "Viscoelastic multilayered blade-type passive vibration isolation system for a spaceborne cryogenic cooler," Cryogenics, vol. 105, p. 102982, 2020.

[17] D. S. Steinberg, Vibration Analysis for Electronic Equipment, John Wiley and Sons, Inc, New York, USA, 3rd edition, 2000.

[18] Innovative Solutions In Space B V, CubeSat solar panels ISIS Innovative Solutions in SpaceJune 2020, https://www.isispace .nl/product/isis-cubesat-solar-panels/.

[19] Innovative Solutions In Space B V, ISIPOD CubeSat deployer ISIS - Innovative Solutions in SpaceJune 2020, https://www .isispace.nl/product/isipod-cubesat-deployer/.

[20] 3M Company, $3 M^{T M}$ adhesive transfer tape 966June 2020, https://www.3m.com/3M/en_US/company-us/all-3mproducts/ /3M-Adhesive-Transfer-Tape966/?N=5002385 $+3293241971 \& \mathrm{rt}=$ rud.

[21] H. U. Oh, H. W. Ha, T. Kim, and J. K. Lee, "Thermo-mechanical design for on-orbit verification of MEMS based solid propellant thruster array through STEP cube lab mission," International Journal of Aeronautical and Space Sciences, vol. 17, no. 4, pp. 526-534, 2016.

[22] S. Tian, Z. Xu, Q. Wu, and C. Qin, "Dimensionless analysis of segmented constrained layer damping treatments with modal strain energy method," Shock and Vibration, vol. 2016, 16 pages, 2016.

[23] T. X. Liu, H. X. Hua, and Z. Zhang, "Robust control of plate vibration via active constrained layer damping," Thin-Walled Structures, vol. 42, no. 3, pp. 427-448, 2004.

[24] A. Torisaka and H. Yamakawa, "Simultaneous optimization of thickness and sticking position of multilayer damping materials for vibration control of small satellite," in 11th AIAA/ISSMO Multidisciplinary Analysis and Optimization Conference, pp. 1633-1646, Portsmouth, Virginia, September 2006.

[25] A. N. Nayak, L. Satpathy, and P. K. Tripathy, "Free vibration characteristics of stiffened plates," International Journal of Advanced Structural Engineering, vol. 10, no. 2, pp. 153-167, 2018.

[26] M. Bilasse, L. Azrar, and E. M. Daya, "Complex modes based numerical analysis of viscoelastic sandwich plates vibrations," Computers \& Structures, vol. 89, no. 7-8, pp. 539-555, 2011.

[27] A. Denis, C. Asma, C. Bernal et al., QB50 system requirements and recommendations, no. 4, pp. 1-28, 2013, June 2020, https://www.qb50.eu.

[28] GEVS: GSFC-STD-7000A, General environmental verification standard (GEVS) for GSFC flight programs and projects, NASA Goddard Space Flight Center, 2013. 